\title{
O uso das redes sociais como elemento de distração nos processos de treinamento
}

El uso de las redes sociales como elemento distractor en procesos de formación

The use of social networks as a distracting element in training processes

\section{Edgar Enrique Villalva Cárdenas}

Magister. Docente Instituto Tecnológico Superior Vicente Rocafuerte, Guayaquil, Ecuador, evillalva@itsvr.edu.ec, https://orcid.org/0000-0003-0664-0292

\section{Rosa Elizabeth Molina Izurieta}

Magister.Docente Instituto Tecnológico Superior Vicente Rocafuerte, Guayaquil, Ecuador, rmolina@itsvr.edu.ec, https://orcid.org/0000-0003-2531-3833

\section{Marco Antonio Villalva Cárdenas}

Magister.Docente Instituto Tecnológico Superior Vicente Rocafuerte, Guayaquil, Ecuador, mvillalva@itsvr.edu.ec, https://orcid.org/0000-0001-7108-5491

Recebido em julho de 2018 - Aceito em novembro de 2018

Formación docente - revista iberoamericana de educación http://www.revista-iberoamericana.org/index.php/es/index

https://creativecommons.org/licenses/by/4.0/deed.es e-ISSN: 2737-632X

Vol - 2 No. 1Janeiro - Março 2019

Pags 27 - 36

Resumo Este trabalho é realizado com o objetivo de conscientizar sobre o impacto da Internet e das redes sociais na prática de crimes contra adolescentes no Equador. Atualmente, a internet e as redes sociais servem como um dos meios de comunicação mais utilizados pelos adolescentes, o que gerou novos hábitos e comportamentos em relação ao uso dessas novas ferramentas. Como a grande maioria dos jovens usa essas tecnologias sem ter conhecimento dos mecanismos de segurança, os criminosos e cibercriminosos modernizaram seus métodos de busca e encontraram possíveis vítimas para cometer seus crimes. Pretende-se conhecer os tipos de crimes cometidos pelas redes sociais de que os adolescentes foram vítimas nos últimos dois anos. O escopo da pesquisa é descritivo e exploratório, 
utilizou-se pesquisa de campo e bibliográfica; foram aplicadas pesquisas a 198 adolescentes entre 12 e 17 anos, selecionados por meio de amostragem por cota e julgamento do pesquisador. gerar informações relevantes em relação aos riscos envolvidos no uso indevido de redes sociais e na prática final de crimes que podem ser realizados utilizando-os como meio de contato.

Palavras-chave: redes sociais; crimes; adolescentes; Internet.

Resumen El presente trabajo se realiza con el propósito de concienciar sobre el impacto del internet y las redes sociales para el cometimiento de delitos en contra de los adolescentes en el Ecuador. En la actualidad el internet y las redes sociales sirven como uno de los medios de comunicación mayormente usados por los adolescentes lo cual ha generado nuevos hábitos $\mathrm{y}$ comportamientos en relación al uso de estas nuevas herramientas. Debido a que los jóvenes en su gran mayoría utilizan estas tecnologías sin tener ningún conocimiento de mecanismos de seguridad, ha provocado que delincuentes y cyber criminales tecnifiquen sus métodos de búsqueda y encuentren posibles víctimas para cometer sus delitos. Se pretende conocer los tipos de delitos cometidos por redes sociales de los cuales han sido víctimas en los últimos dos años los adolescentes. El alcance de la investigación es de tipo descriptiva y exploratoria, se usó investigación de campo y bibliográfico, se aplicó encuestas a 198 adolescentes comprendidos entre 12 y 17 años los cuales fueron seleccionados mediante el uso del muestreo por cuota y juicio del investigador La investigación pretende generar información relevante en relación a los riesgos que conlleva la mala utilización de las redes sociales y el final cometimiento de delitos que se pueden realizar usándolas como un medio de contacto.

Palabras clave: redes sociales; delitos; adolescentes; internet. 
Abstract. The present work is carried out with the purpose of raising awareness about the impact of the internet and social networks for committing crimes against adolescents in Ecuador. Currently the internet and social networks serve as one of the means of communication mostly used by adolescents which has generated new habits and behaviors in relation to the use of these new tools. Because the majority of young people use these technologies without having any knowledge of security mechanisms, it has caused criminals and cyber criminals to technify their search methods and find possible victims to commit their crimes. It is intended to know the types of crimes committed by social networks of which they have been victims in the last two years,. The scope of the research is descriptive and exploratory, field and bibliographical research was used, surveys were applied to 198 adolescents aged between 12 and 17 years who were selected through the use of quota sampling and investigator judgment. generate relevant information in relation to the risks involved in the misuse of social networks and the final committing of crimes that can be carried out using them as a means of contact. Keywords: social networks; crimes; teenagers; Internet.

\section{INTRODUÇÃO}

Nos últimos tempos, pornografia infantil, fraudes eletrônicas e até atividades terroristas têm sido consideradas como novos crimes de computador, todos os quais atentam contra a liberdade, a integridade e a dignidade das pessoas. Crimes desse tipo são atividades ilegais cometidas por meios e dispositivos tecnológicos e de comunicação, ações como gravações e fotografias sem consentimento ou autorização legal, representação de senhas eletrônicas, danos, perda de informações intencionais, intervenção ou violação. Para a privacidade das pessoas, elas são realizadas com o objetivo de causar alguns 
danos, causar perdas pessoais e institucionais ou impedir o uso de sistemas de computador.

Entre um dos métodos para cometer crimes através do uso da Internet em escala global, poderíamos dizer que certas pessoas aproveitam essas tecnologias e modernizam seus métodos de busca e captura de possíveis vítimas por meio de redes sociais, chats, mecanismos de busca parceiros e publicação. de ofertas de emprego ou bolsas falsas, permitindo que eles obtenham informações de contato para persuadir, capturar e sujeitá-las posteriormente ao tráfico de pessoas. É muito vantajoso para os criminosos usar esses meios de comunicação porque eles têm a possibilidade de se passar por outra pessoa, conhecer pessoas de diferentes partes do mundo e selecionar as características de suas possíveis vítimas por meio de pesquisas avançadas de informações.

Sendo o país um país de alta mobilidade migratória de origem, trânsito e destino de pessoas, muitos deles foram vítimas de tráfico de pessoas e, principalmente, mulheres, adolescentes e crianças, vítimas de tráfico e exploração sexual. trabalho [6]. As vítimas podem ser contatadas no Equador e exploradas em outros países (tráfico internacional) ou transferidas de uma área do Equador para outra (tráfico interno).

No Equador, esse tipo de crime é tipificado no atual Código Penal Orgânico Integral, em sua segunda seção, tráfico de pessoas, artigo 91, "O recrutamento, transporte, transferência, entrega, recepção ou recepção para si ou para terceiros, de uma ou mais pessoas, dentro do país ou de ou para outros países para fins de exploração, constituem crime de tráfico de pessoas "e, de acordo com o artigo 92, a sanção imposta pelo Estado àqueles que incorrem nesse crime é a penalidade exclusiva de liberdade entre treze a vinte e seis anos, de acordo com sua circunstância agravante [7]. 
Nas investigações realizadas pela Procuradoria Geral da República, constatou-se que em muitos casos a forma de contato com as vítimas se dá pela internet e redes sociais, através da publicidade enganosa de supostas ofertas de emprego, convites para festas de adolescentes, promoção de bolsas de estudos no exterior ou paixões falsas. Todas essas estratégias foram usadas pelo traficante para enganar a vítima [8].

Por se tratar de um problema social que envolve todos nós, é necessário conscientizar a sociedade e, principalmente, os adolescentes mais vulneráveis nesse tipo de crime e promover mecanismos de prevenção e segurança em relação às informações publicadas na mídia eletrônica. .

Também é responsabilidade do Estado equatoriano garantir o cumprimento de um dos direitos básicos estabelecidos na Constituição, como o direito à liberdade, indicado no artigo 66, número 29, alínea b) literal) "o reconhecimento de que todas as pessoas nascem livres e, portanto, a proibição de todas as formas de escravidão, exploração, servidão e tráfico de seres humanos em todas as suas formas "[3].

A presente investigação tenta identificar os tipos de crimes cometidos contra adolescentes por meio de redes sociais na cidade de Guayaquil-Equador, explicar por que os adolescentes preferem usar as redes sociais como um meio de comunicação em vez dos meios tradicionais, e sugerir de maneira técnica os tipos de controles que os pais devem aplicar aos filhos em relação ao uso da Internet e das redes sociais.

Além disso, o estudo tem como objetivo responder às seguintes perguntas:

- Maior controle dos pais sobre o uso das redes sociais, reduzindo o risco de os adolescentes serem vítimas de crimes cometidos nas redes sociais.

- Saiba em que idade os adolescentes começaram a usar algum tipo de rede social. 


\section{MATERIAIS E MÉTODOS}

Esta pesquisa é descritiva, quantitativa e metodológica, destinada a adolescentes entre 12 e 17 anos na cidade de Guayaquil, foram pesquisados 198 jovens, dos quais 193, ou seja, 97,47\% indicaram ser usuário das redes sociais, que foram questionadas sobre diferentes tópicos relacionados ao tópico em questão. Sua seleção foi através do uso de amostragem de cota e julgamento do investigador.

Também é uma investigação documental, porque os dados oficiais fornecidos pela Unidade Especializada em Crime de Computador foram coletados no Ministério Público do Cantão de Guayaquil, província de Guayas, com base nas denúncias recebidas de infrações cometidas pelo uso de redes sociais a adolescentes entre 12 e 17 anos. que viveram no Equador durante os anos 2017-2018.

Para o processamento das informações, utilizou-se estatística descritiva, os

dados foram registrados e analisados, gerando gráficos e tabelas que permitiram obter uma distribuição de frequências, o que permitiu analisar os resultados obtidos de forma simples e clara.

Os dados da pesquisa foram coletados pela técnica de pesquisa, aplicada apenas uma vez. As informações também foram obtidas nas denúncias registradas no Ministério Público.

Para a análise quantitativa dos dados da pesquisa, foi utilizado o pacote estatístico SPSS versão 17.0 para Windows.

\section{RESULTADOS}

As seguintes informações foram obtidas das pesquisas realizadas com os adolescentes que fizeram parte da amostra: 46,97\% dos entrevistados eram mulheres e 53,04\% eram homens. Verificou-se que a rede social mais 
utilizada é o Facebook com 84,46\% de aceitação, seguido pelo WhatsApp com $73,06 \%$ e Instagram com $60,10 \%$, o que é evidenciado na tabela a seguir: Evidenciou-se que $30,81 \%$ da amostra foi vítima de algum tipo de crime pelas redes sociais. $69,19 \%$ dos afetados indicaram que não foram prejudicados de forma alguma por meios de computador. A tabela a seguir mostra os tipos de crimes dos quais os adolescentes pesquisados que indicaram ter sido vítimas de um crime por meio de redes sociais foram vítimas. Observa-se que o maior crime cometido são ameaças com $34,69 \%$, seguido de roubo de identidade com $10,26 \%$, contato para fins sexuais $13,23 \%$, pornografia $27,79 \%$, divulgação de fotos íntimas $14,03 \%$ e crimes de conteúdo sexual. Na tabela anterior, observa-se que há muito pouco controle dos pais nas atividades realizadas pelos filhos nas redes sociais, apenas $12,82 \%$ costumam checar as contas das redes sociais dos filhos.

\section{CONCLUSÕES}

A partir da análise realizada, pode-se concluir que, apesar das ameaças das redes sociais serem o crime mais frequentemente cometido contra adolescentes de acordo com as pesquisas, este não aparece como o maior índice de denúncias na acusação, sendo o contato para fins sexuais o crime mais denunciado. Pornografia e tráfico de pessoas é um crime que viola os direitos humanos das vítimas, sendo um dos problemas de segurança mais complicados do país. É necessário tratamento especial, devido à sua complexidade; porque diferentes formas de crime são combinadas, como pornografia, sequestro e, finalmente, exploração de pessoas. Todas essas desvantagens dificultam o manuseio do crime, as vítimas de medo ou ignorância não têm acesso às reclamações, reconhecimento e proteção das vítimas. Percebe-se que os pais ou responsáveis têm muito pouco controle sobre as atividades realizadas pelos adolescentes nas redes sociais, que 
acessam essas mídias sem nenhum tipo de vigilância, motivo pelo qual são suscetíveis de serem vítimas de crimes por esses meios.

\section{REFERÊNCIAS}

Abhishek Kumar, Subham Kumar Gupta, Animesh Kumar Rai, and Sapna Sinha, 2013. Social Networking Sites and Their Security Issues. International Journal of Scientific and Research Publications.

Elisa Bertino, James Caverlee, and Elena Ferrari, 2014. Identity, Privacy, and Deception in Social Networks. Published by the IEEE Computer Society. www.computer.org/internet/.

Barros-Bastidas, C., \& Turpo, O. (2020). La formación en investigación y su incidencia en la producción científica del profesorado de educa- ción de una universidad pública de Ecuador. Publicaciones, 50(2), 167-185. doi:10.30827/publicaciones.v50i2.13952

Barros Bastidas, C., \& Turpo Gebera, O. (2018). Factors influencing the scientific production of university professors: a systematic review . Pensamiento Americano, 11(22). https://doi.org/10.21803/pensam.v11i21-1.276

Constitución de la República del Ecuador, 2008. Asamblea Constituyente. Registro Oficial 449. Ecuador.

Antonio García Jiménez, Pilar Beltrán Orenes, y Carmen Pérez Pais, 2011. La Investigación sobre los Usos y Riesgos de Internet en Menores y Jóvenes. Estado de la Cuestión en España y Proyección Iberoamericana. Confederación Inberoamericana de Asociaciones Científicas y Académicas de la Comunicación.

Michael Fire, Roy Goldschmidt, and Yuval Elovici, 2014. Online Social Networks: Threats and Solutiones. IEEE Communication Survey \& Tutorials. 
Edgar Andrés Buitrón, 2011. Estudio sobre el Estado de la Trata de Personas en Ecuador. Programa de Fortalecimiento de Capacidades para Combatir el Crimen ACCBP, del Ministerio de Asuntos Exteriores y Comercio Internacional del Gobierno de Canadá. Lima, Perú.

Código Orgánico Integral Penal, 2014. Asamblea Nacional del Ecuador. Registro Oficial No. 180. Ecuador.

Fiscalía General del Estado Ecuador. Revista Fiscalía Ciudadana, No. 27. La víctima de trata, un objeto para comprar y vender.

von Feigenblatt, Otto Federico, Costa Rica's Foreign Policy: Can 'Right' Become 'Might'? (November 27, 2008). Journal of Alternative Perspectives in the Social Sciences, Vol. 1, No. 1, pp. 11-15, 2008, Available at SSRN: https://ssrn.com/abstract=1308245

von Feigenblatt, Otto Federico, Human Security and the Responsibility to Protect: A Holistic Approach to Dealing with Violent Conflict in Southeast Asia (May 13, 2009). Journal of Social Sciences, Vol. 11, No. $1, \quad$ pp. 27-40, 2009, Available at SSRN: $\underline{\text { https://ssrn.com/abstract }=1570171}$

von Feigenblatt, Otto Federico, The Importance of Culture in Emic Interpretations of the History of Thailand's Southern Separatist Movement: The 'Gentlemen's Agreement' of 1943 and the Relationship of Malaysia with the Separatists (October 9, 2009). Ritsumeikan Center for Asia Pacific Studies Working Paper No. 097 , Available at SSRN: https://ssrn.com/abstract=1486224 or http://dx.doi.org/10.213 9/ssrn.1486224http://issuu.com/fiscaliaecuador/docs/revista_fiscalia ciudadana_nro 27 
